\title{
7 How Does Visual Attention Differ Between Experts and SUPER \\ Novices on Physics Problems?
}

Adrian Carmichael, Adam Larson, Elizabeth Gire, Lester Loschky \& N. Sanjay Rebello, Kansas State University

\section{OBJECTIVE}

Investigate how the allocation of visual attention differs between experts and novices on physics problems where the critical information needed to answer the problem is contained in a diagram.

\section{INTRODUCTION}

\section{Previous Research}

- Experts' visual attention is primarily driven by knowledge and they spend more time than novices looking at relevant information in figures. [1-3]

- Novices' visual attention is driven by noticeable features of environment and they spend more time looking at perceptually salient areas of figures and pictures. [4]

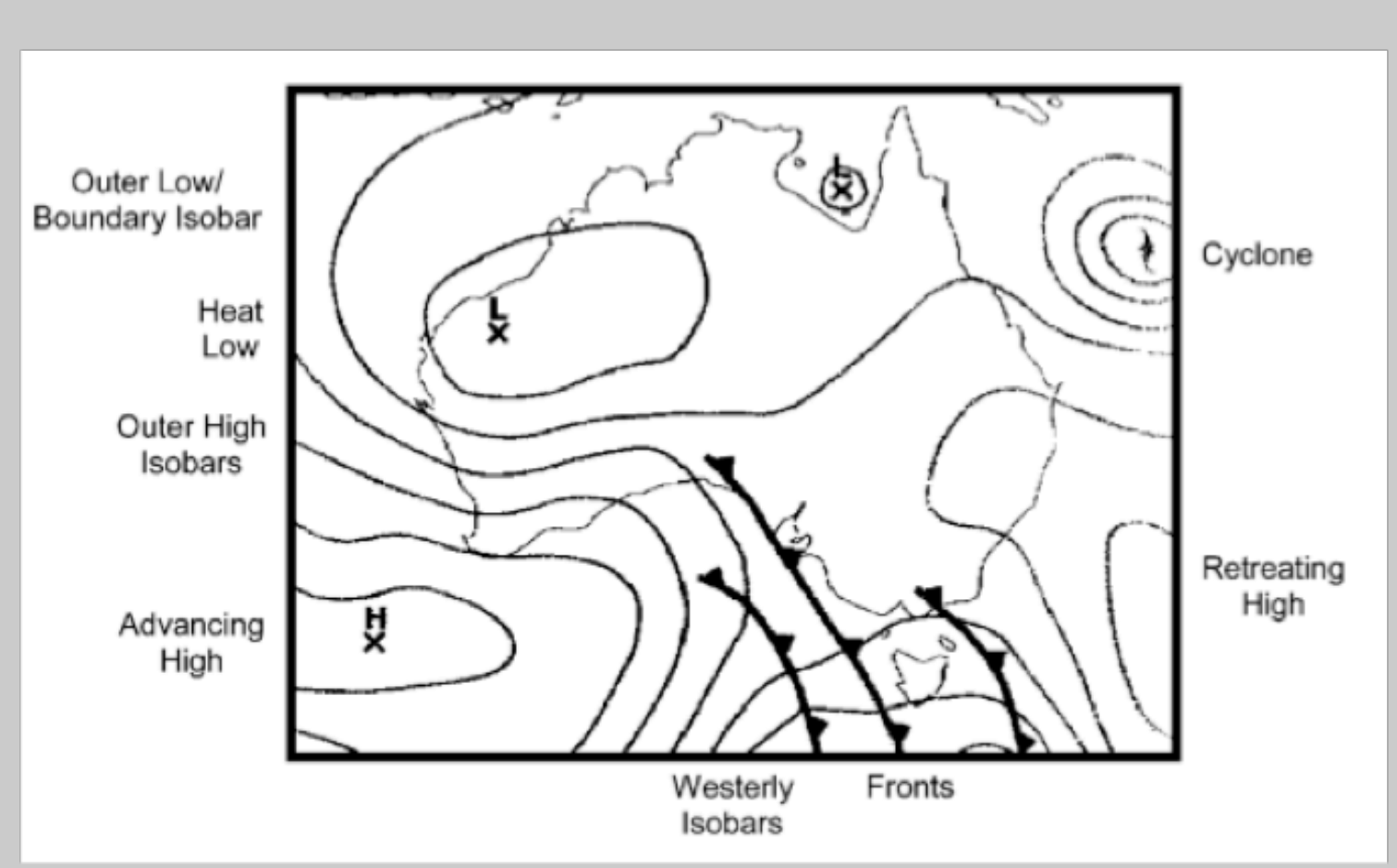

Novices were found to spend more time looking at salient features of weather map. [3]

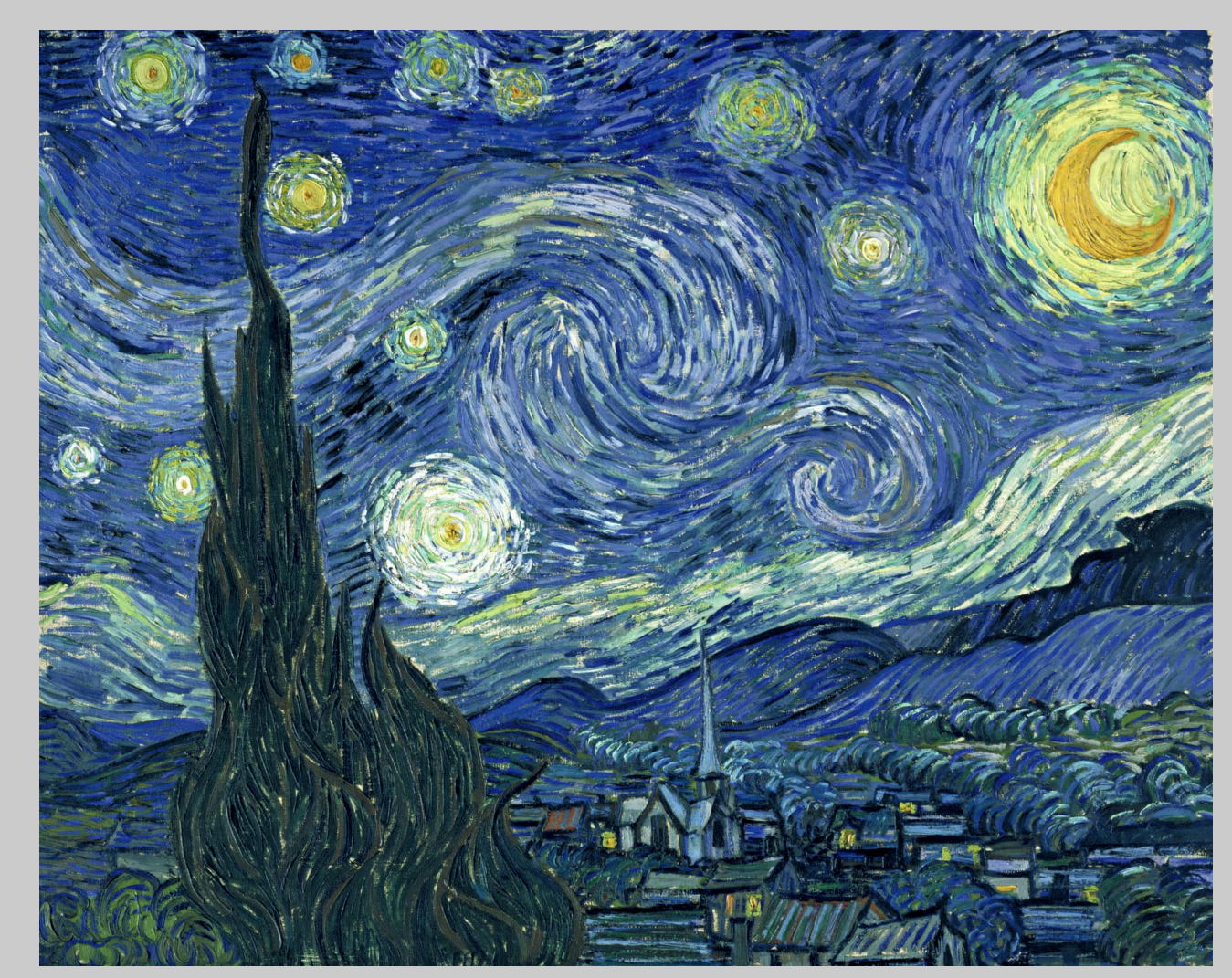

Artists spend more time looking at relevant areas of paintings than nonartists. [2]

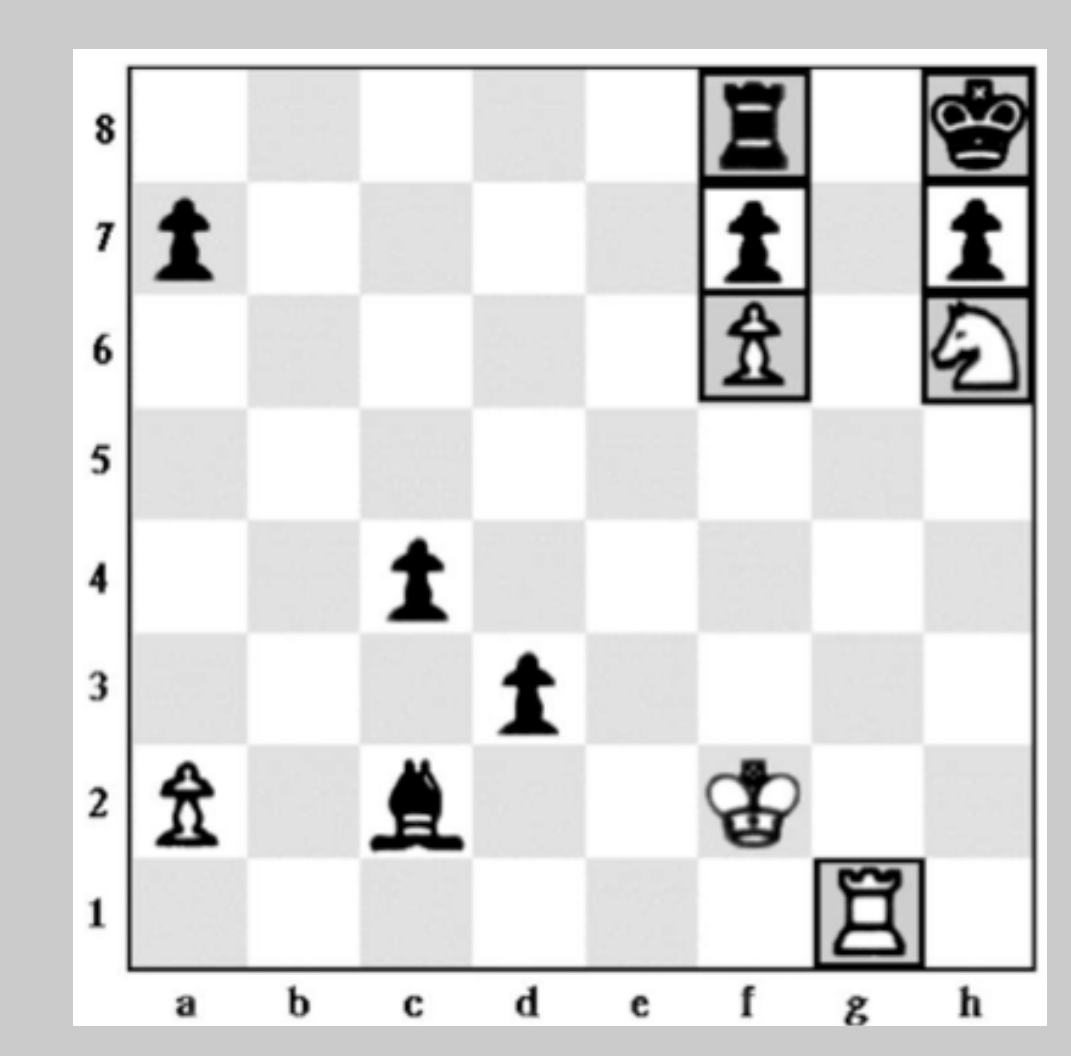

Expert chess players have higher densities of fixations on relevant chess

Motivation: If similar differences in visual attention exist for physics problems, guiding novices' eye movements to match those of experts may be helpful.

\section{Research Question}

How does expertise affect the dwell time in perceptually salient versus thematically relevant areas in a figure?

Perceptually salient: most noticeable portions of a diagram or picture

Thematically relevant: portions of a diagram which contain necessary information Dwell time: total time spent looking at an area while viewing the diagram

\section{METHOD}

Participants: 9 PhD students in physics with teaching experience and 13 introductory psychology students who have taken a physics course.

Physics Problems: Participants answered 10 multiple-choice conceptual physics questions where the information needed to answer the question was contained in a diagram.

Eye Tracking: Eye movements were recorded with an EyeLink 1000 eye tracker.

Instructions and calibration of eye tracker

Answer 10 multiple-choice 2 conceptual questions while eye movements recorded

Explain reasoning for answers to questions while watching playback of eye movements
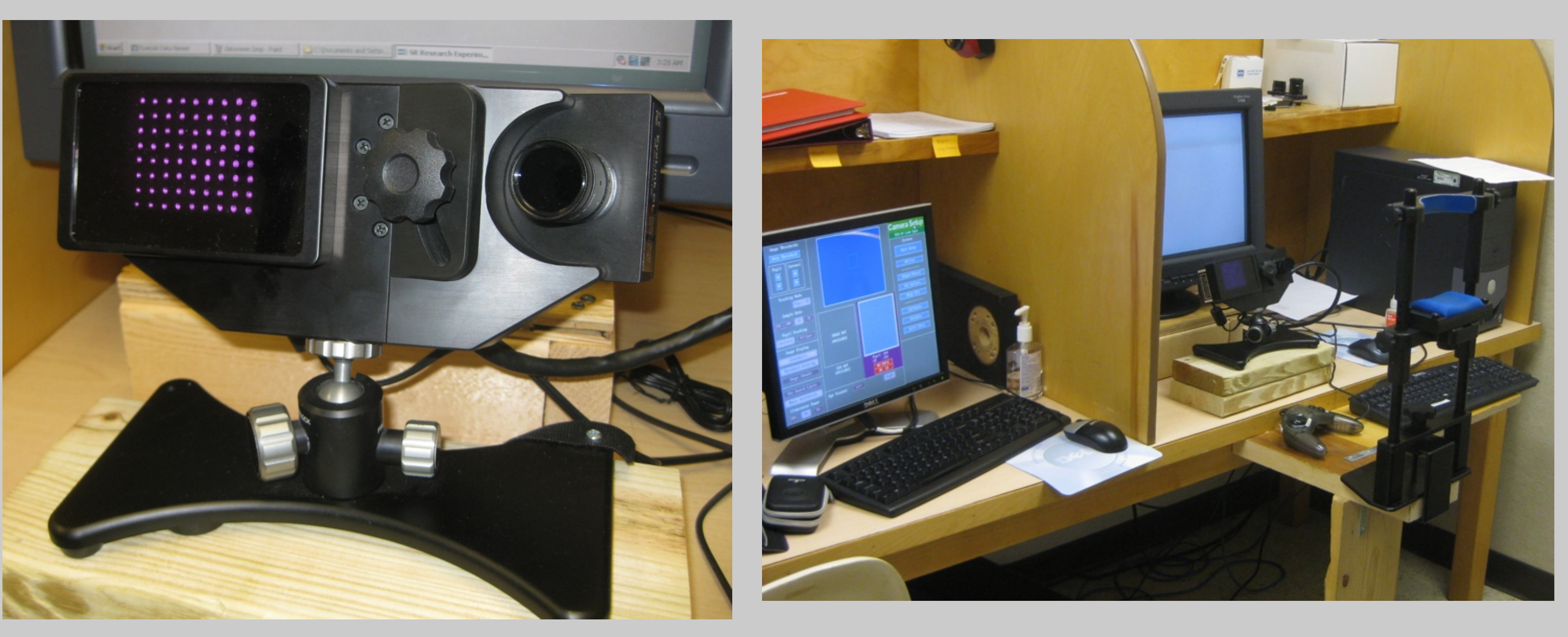

EyeLink 1000 (left) and data collection room (right)

\section{ANALYSIS \& RESULTS}

- Perceptually salient and thematically relevant areas of interest (AOI's) defined by three independent raters.

- One-way ANOVA used to compare percentage of time spent in each type of AOI. -Independent variable: correctness of answer

${ }^{*}$ Significance determined at alpha $=.05$ level. Green boxes indicate significant differences.

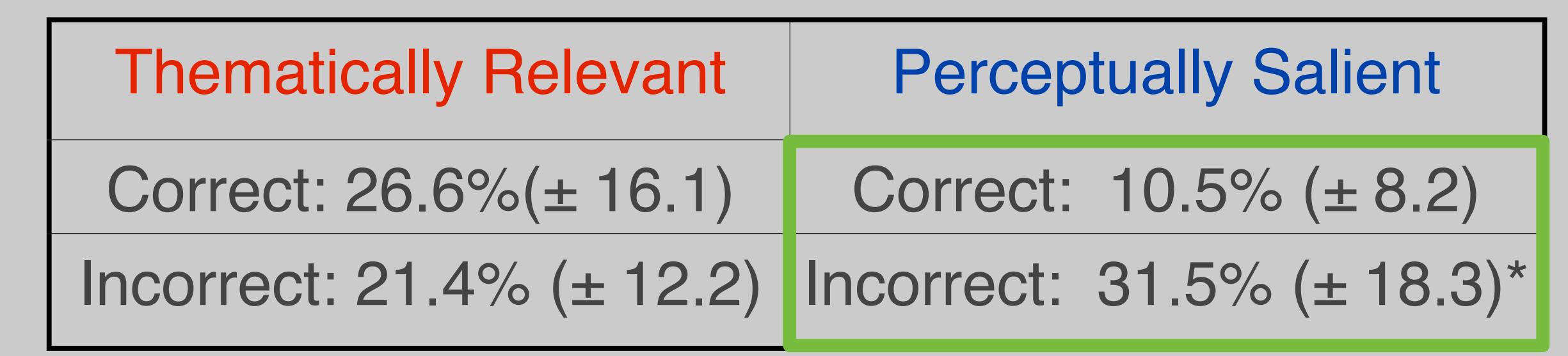

\begin{tabular}{|c|c|}
\hline Thematically Relevant & Perceptually Salient \\
\hline Correct: $46.6 \%( \pm 10.7)$ & Correct: $19.2 \%( \pm 8.2)$ \\
\hline Incorrect: $25.8 \%( \pm 11.5)^{*}$ & Incorrect: $29.0 \%\left( \pm 6.9^{*}\right.$ \\
\hline
\end{tabular}
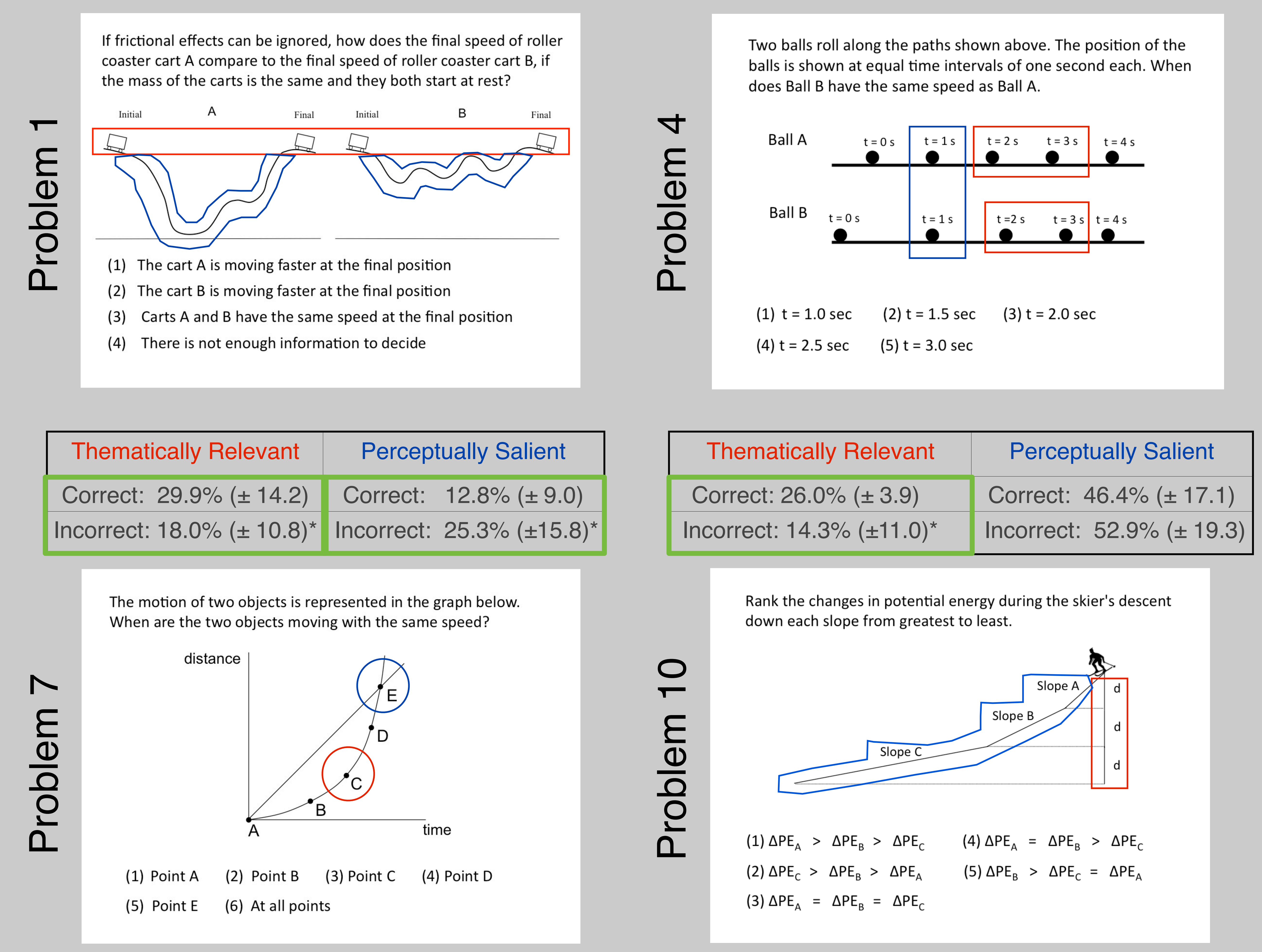

\section{CONCLUSION}

- Found significant differences in the way those who answered correctly versus incorrectly allocated visual attention on physics problems about energy and speed.

- Provides some evidence to support previous findings:

Those who answer correctly spend more time looking at thematically relevant elements.

Those who answer incorrectly spend more time looking at perceptually salient portions.

- Lays the foundation for future work in guiding novices' attention to mimic that of experts using visual cueing techniques.

REFERENCES

1. T. van Gog, H. Jarodzka, K. Scheiter, P. Gerjets \& F. Paas, Computers in Human Behavior 25, 785-791 (2009). 2. J. R. Antes \& A. F. Kristjanson, Perceptual and Motor Skills 73, 893-894 (1991).

3. N. Charness, E. M. Reingold, M. Pomplun \& D.M. Stampe, Memory and Cognition 29, 1146-1152 (2001). 4. R.K. Lowe, Learning and Instruction 12, 157-176 (2003). 